\title{
Neuropeptide FF increases M2 activation and self-renewal of adipose tissue macrophages
}

\author{
Syed F. Hassnain Waqas, ${ }^{1}$ Anh Cuong Hoang, ${ }^{1}$ Ya-Tin Lin, ${ }^{2}$ Grace Ampem, ${ }^{1}$ Hind Azegrouz, ${ }^{3}$ Lajos Balogh, ${ }^{4}$ Julianna Thuróczy, ${ }^{5}$ \\ Jin-Chung Chen, ${ }^{2}$ Ivan C. Gerling, ${ }^{6}$ Sorim Nam, ${ }^{7}$ Jong-Seok Lim, ${ }^{7}$ Juncal Martinez-Ibañez, ${ }^{8}$ José T. Real, ${ }^{8}$ Stephan Paschke, ${ }^{9}$ \\ Raphaëlle Quillet, ${ }^{10}$ Safia Ayachi, ${ }^{10}$ Frédéric Simonin, ${ }^{10}$ E. Marion Schneider, ${ }^{11}$ Jacqueline A. Brinkman, ${ }^{12,13}$ Dudley W. Lamming, ${ }^{12,13}$ \\ Christine M. Seroogy, ${ }^{12}$ and Tamás Röszer ${ }^{1}$ \\ Institute of Comparative Molecular Endocrinology, University of Ulm, Ulm, Germany. 2Department of Physiology and Pharmacology and Graduate Institute of Biomedical Sciences, Chang Gung University; \\ Neuroscience Research Center, Chang Gung Memorial Hospital, Taoyuan, Taiwan. ${ }^{3}$ Massachusetts Institute of Technology, Cambridge, Massachusetts, USA. ${ }^{4}$ National Research Institute for Radiobiology and \\ Radiohygiene, Budapest, Hungary. ${ }^{5}$ University of Veterinary Medicine, Budapest, Hungary. ${ }^{6}$ Department of Medicine, University of Tennessee, Memphis, Tennessee, USA. 'Department of Biological Science, \\ Sookmyung Women's University, Seoul, South Korea. ${ }^{8}$ Department of Medicine, Hospital Clínico Universitario de València, Centro de Investigación Biomédica en Red de Diabetes y Enfermedades Metabólicas \\ Asociadas (CIBERDEM), Valencia, Spain. ${ }^{9}$ Department of General and Visceral Surgery, University Hospital Ulm, Ulm, Germany. ${ }^{10}$ Biotechnologie et Signalisation Cellulaire, UMR 7242 , Centre National de \\ Recherche Scientifique (CNRS), Université de Strasbourg, IIlkirch, France. "'Division of Experimental Anesthesiology, University Hospital Ulm, Ulm, Germany. ${ }^{12 U n i v e r s i t y ~ o f ~ W i s c o n s i n, ~ S c h o o l ~ o f ~ M e d i c i n e ~ a n d ~}$ \\ Public Health, Madison, Wisconsin, USA. ${ }^{13}$ William S. Middleton Memorial Veterans Hospital, Madison, Wisconsin, USA.
}

\begin{abstract}
The quantity and activation state of adipose tissue macrophages (ATMs) impact the development of obesity-induced metabolic diseases. Appetite-controlling hormones play key roles in obesity; however, our understanding of their effects on ATMs is limited. Here, we have shown that human and mouse ATMs express NPFFR2, a receptor for the appetite-reducing neuropeptide FF (NPFF), and that NPFFR2 expression is upregulated by IL-4, an M2-polarizing cytokine. Plasma levels of NPFF decreased in obese patients and high-fat diet-fed mice and increased following caloric restriction. NPFF promoted M2 activation and increased the proliferation of murine and human ATMs. Both M2 activation and increased ATM proliferation were abolished in NPFFR2-deficient ATMs. Mechanistically, the effects of NPFF involved the suppression of E3 ubiquitin ligase RNF128 expression, resulting in enhanced stability of phosphorylated STAT6 and increased transcription of the M2 macrophage-associated genes IL-4 receptor $\alpha$ (I/4ra), arginase 1 (Arg1), IL-10 (I/10), and alkylglycerol monooxygenase (Agmo). NPFF induced ATM proliferation concomitantly with the increase in N-Myc downstream-regulated gene 2 (Ndrg2) expression and suppressed the transcription of Ifi200 cell-cycle inhibitor family members and MAF bZIP transcription factor B (Mafb), a negative regulator of macrophage proliferation. NPFF thus plays an important role in supporting healthy adipose tissue via the maintenance of metabolically beneficial ATMs.
\end{abstract}

\section{Introduction}

Adipose tissue macrophages (ATMs) are immune cells of adipose tissue (AT) stroma with key roles in AT development and metabolism (1). ATMs accumulate in AT in obesity and undergo so-called classical M1 polarization to a metabolically harmful proinflammatory phenotype, which drives obesity-associated metabolic diseases (2). M1-activated ATMs produce inflammatory cytokines, NO, and ROS that induce AT inflammation, leading to insulin resistance (IR) and contributing to comorbidities of obesity (2). Given the near-pandemic incidence of obesity and associated metabolic diseases, there is a clear need for a better understanding of how the quantity and activation of ATMs are controlled (1).

M1-activated ATMs are replenished from circulating monocytes that infiltrate obese AT (3). ATMs also proliferate locally in obese AT, increasing the quantity of M1-activated ATMs and exacerbating AT inflammation (4). ATMs can also adopt an alternative

Conflict of interest: The authors have declared that no conflict of interest exists. Submitted: August 31, 2016; Accepted: April 6, 2017

Reference information: J Clin Invest. 2017;127(7):2842-2854.

https://doi.org/10.1172/JCI90152 or M2 activation state, which supports the resolution of inflammation and is hence metabolically beneficial; the prevalent ATM activation state in lean AT is M2 (2). Th2 cytokines, lipid metabolites, neurotransmitters, and hormones can shift ATMs into an M2activated state (5); however, how these signals function to control the replenishment of metabolically beneficial ATMs is unknown.

Neuropeptide FF (FLFQPQRFamide, abbreviated herein as $\mathrm{NPFF}$ ) is a member of the evolutionarily conserved RF-amide neuropeptide family (6). NPFF has been principally studied in mammals as a pain modulator and in the adaptive responses to opiate treatment, but it can also reduce food intake in rodents by acting at hypothalamic nuclei $(6,7)$. Appetite control is key in obesity development, and in vitro studies have shown that NPFF inhibits both human and murine adipocyte development $(8,9)$. Peripheral tissues have negligible NPFF receptor expression, with the exception of AT, in which NPFF receptor 2 (NPFFR2, also known as GPR74, HG31, and NPFF2) is expressed $(10,11)$. Genetic variance of NPFFR2 is closely associated with obesity status (12). Human plasma contains NPFF (13), and it is postulated that NPFF may be released from nerve endings in AT (11). However, the in vivo role of NPFF in AT function is unknown. 
A

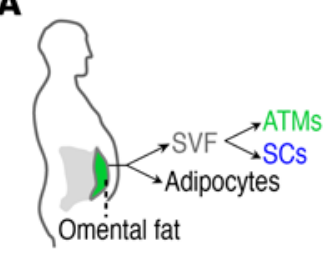

$\mathbf{F}$

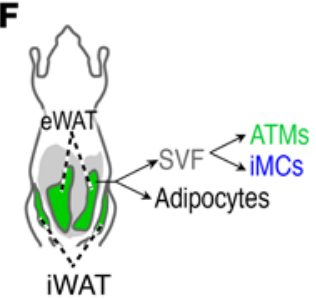

K

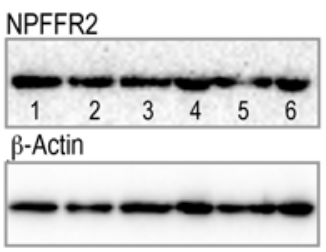

B
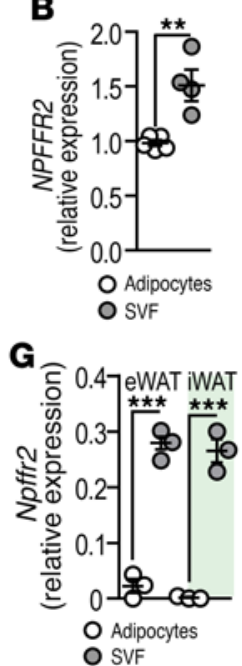

L

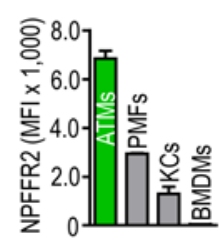

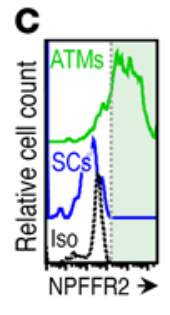
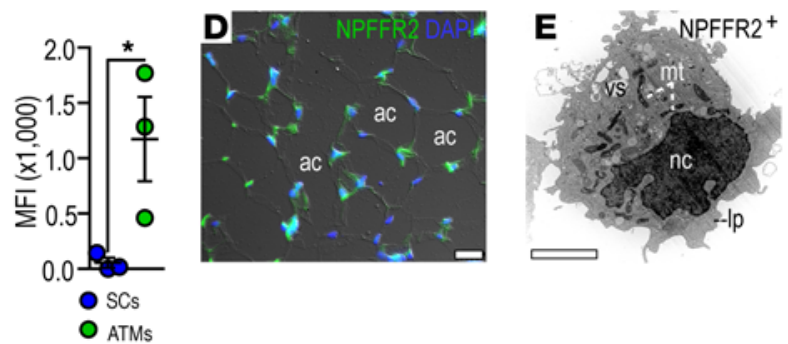

H

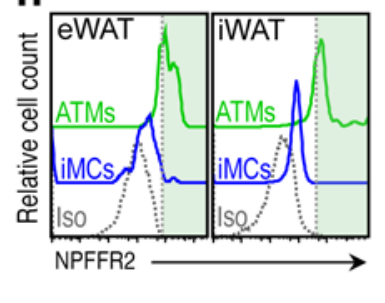

I
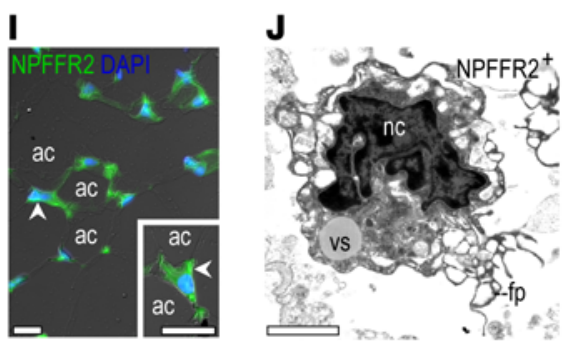

M

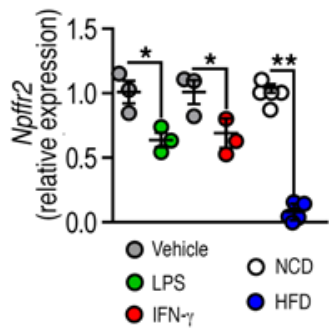

N

0

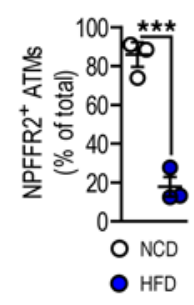

$\mathbf{P}$

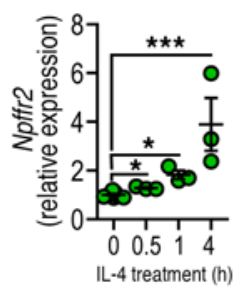

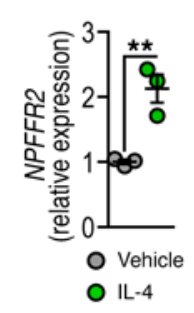

Figure 1. Human and mouse ATMs express NPFFR2, which increases after IL-4 exposure. (A) Diagram of human cell types of the omental fat depot analyzed in this study. ATMs, CD68 ${ }^{+}$CD14+ ATMs; SCs, CD68-CD14- stromal cells. (B) NPFFR2 transcription in SVF and adipocytes of human omental fat $(n=4)$. (C) FACS analysis of NPFFR2 expression in human ATMs and SCs. (D) NPFFR2 immunostaining in human omental fat. Scale bar: $25 \mu \mathrm{m}$. (E) TEM image of an NPFFR2 ${ }^{+}$ human ATM. Scale bar: $0.5 \mu \mathrm{m}$. (F) Diagram of the analyzed mouse fat depots. (G) Relative transcription of Npffr 2 in SVF of lean mice; each data point shows pools of cells from 3 mice. (H) FACS analysis of NPFFR2 expression in mouse ATMs and iMCs. (I) Immunostaining of NPFFR2 in mouse eWAT. Arrowheads indicate the cell border. Scale bars for both images: $25 \mu \mathrm{m}$. (J) TEM image of an NPFFR2 ${ }^{+}$mouse ATM. Scale bar: $0.5 \mu \mathrm{m}$. (K) Western blot of NPFFR2 in mouse ATMs. Lanes 1-3: ATMs from eWAT; lanes 4-6: ATMs from iWAT. (L) FACS analysis of NPFFR2 expression in mouse macrophages. PMFs, peritoneal macrophages; KCs, Kupffer cells; BMDMs, nonstimulated bone marrow-derived macrophages $(n=3)$. (M) Npffr2 transcription in mouse ATMs after $100 \mathrm{ng} / \mathrm{ml}$ LPS or $2.5 \mathrm{ng} / \mathrm{ml} \mathrm{IFN}-\gamma$ treatment for 4 hours in vitro or after 3 months on a NCD or HFD ( $n=3$ and 5 , respectively). (N) NPFFR2 ${ }^{+}$ATMs in NCD- or HFD-fed mice. Each data points represent pooled ATMs from 2 mice. The experiment was conducted 2 times. ( $\mathbf{O}$ and $\mathbf{P}) \mathrm{Npffr} 2$ and NPFFR2 transcription after $10 \mathrm{ng} / \mathrm{ml} \mathrm{lL}-4$ treatment of mouse $(\mathbf{O})$ and human $(\mathbf{P})$ ATMs $(n=3)$. Human ATMs were treated for 4 hours. ${ }^{*} P<0.05,{ }^{* *} P<0.01$, and ${ }^{* * *} P<0.001$, by unpaired, 2-tailed Student's $t$ test (B, C, G, M, N and $\mathbf{P}$ ) and 1-way ANOVA with Dunnett's post-hoc test (0). ac, adipocyte; fp, filopodia; iMCs, immature myeloid cells; iso, isotype; Ip, lamellipodia; MFI, mean fluorescence intensity; mt, mitochondria; $\mathrm{nc}$, nucleus; vs, vesicle.

The mechanisms governing macrophage proliferation and M1/M2 polarization are key to defining the metabolic effects of ATMs (2). We show here that NPFF has metabolically beneficial effects on ATMs by increasing M2-macrophage activation and proliferation. Thus, NPFF appears to play an important role in the maintenance of healthy AT.

\section{Results}

NPFF receptor expression is restricted to ATMs in AT, and its expression is upregulated by $I L-4$. NPFFR 2 is the principal receptor for NPFF in mammalian cells, and its gene expression has been demonstrated in AT (10). To study the cell-autonomous function of NPFFR2 in AT, we first examined its expression in human omental fat (Figure 1A). We found that NPFFR2 mRNA transcription and NPFFR2 protein expression were restricted to ATMs in the stromal vascular fraction (SVF) (Figure 1, B-E; details of ATM isolation are provided in Supplemental Figure 1; supplemental material available online with this article; https://doi.org/10.1172/JCI90152DS1). Like human AT, we observed that Npffr2 mRNA and NPFFR2 protein were expressed only by ATMs in mouse epididymal white adipose tissue (eWAT) and inguinal WAT (iWAT) (Figure 1, F-J). The expression of NPFFR2 was equivalent in ATMs of eWAT and iWAT (Figure 1K). Because eWAT from lean mice contained more ATMs than did iWAT (Supplemental Figure 1, B and C), we used ATMs isolated from eWAT for in vitro assays throughout the study. Other potential receptors of NPFF, such as Npffr1 and acid-sensing ion channel 1 (Asic1a), were not expressed by ATMs (Supplemental Figure 2, A and B).

We next compared NPFFR2 expression levels between ATMs and other macrophage types in mice. Peritoneal macrophages, Kupffer cells, and bone marrow-derived macrophages had minor or negligible NPFFR2 protein and Npffr2 mRNA expression levels relative to levels detected in ATMs (Figure 1L and Supplemental Figure 2, C and D), whereas the murine macrophage line J774A.1 had more detectable expression of Npffr2 (Supplemental Figure $2 \mathrm{E})$. Moreover, NPFFR2 transcription was undetectable in human peripheral blood monocytes and in nonadherent, monocytic THP1 

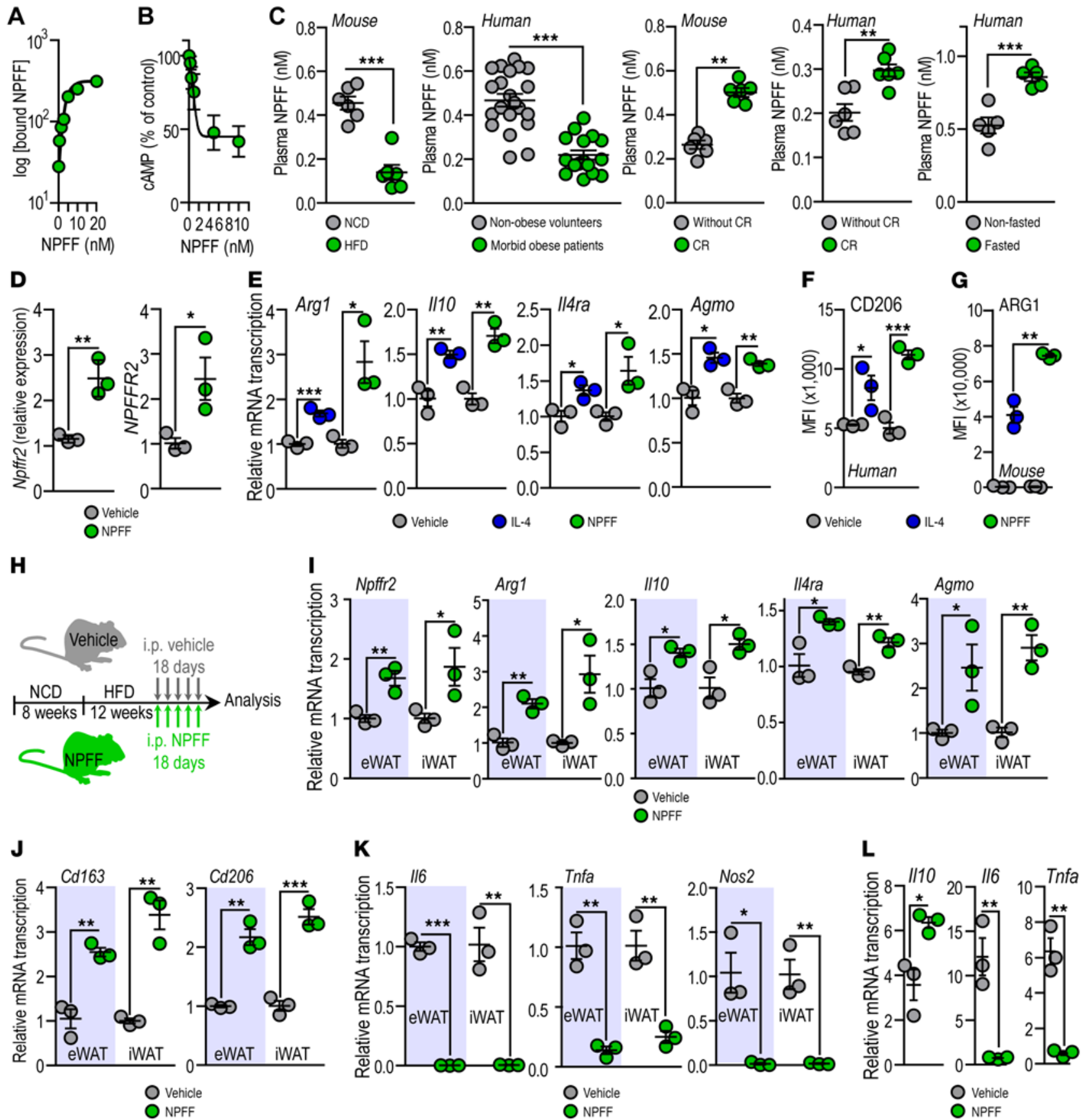

Figure 2. Human and mouse plasma contains sufficient NPFF to provoke M2 activation of ATMs. (A) Binding of FITC-conjugated NPFF to mouse ATMs. (B) CAMP levels in mouse ATMs treated with NPFF. (C) Plasma NPFF levels were measured in NCD- and HFD-fed mice $(n=6)$; in nonobese volunteers ( $n=21)$ and morbidly obese patients $(n=16)$; in obese mice without or with 14 days of CR $(n=6)$; in morbidly obese patients with or without a CR diet $(n=6)$; and in nonobese, nonfasted volunteers and nonobese volunteers following 28 days of intermittent fasting $(n=5)$. (D) Npffr2 and NPFFR2 transcription in response to treatment of mouse and human ATMs for 4 hours with $0.5 \mathrm{nM} \mathrm{NPFF}(n=3)$. (E) Transcription of M2 macrophage activation genes following a 30-minute treatment of mouse ATMs with $10 \mathrm{ng} / \mathrm{ml} \mathrm{IL-4}$ or $0.5 \mathrm{nM}$ NPFF. (F) FACS analysis of human ATMs treated with $0.5 \mathrm{nM}$ NPFF for 4 hours. Each data point represents pooled ATMs from 2 donors. (G) Arginase 1 (ARG1) expression of mouse ATMs treated with $10 \mathrm{ng} / \mathrm{ml} \mathrm{IL-4}$ or $0.5 \mathrm{nM} \mathrm{NPFF}$ for 24 hours. Each data point represents pooled ATMs from 3 to 5 mice. (H) Scheme of NPFF treatment of HFD-fed mice. (I-K) Relative transcription of Npffr2, M2, and M1 genes in ATMs from HFD-fed mice, without or with NPFF treatment ( $n=6$; each point represents pooled ATMs from 2 mice). (L) Effect of NPFF on palmitic acid-treated ATMs. ATMs were pooled from 3 to 5 mice and treated in triplicate in panels $\mathbf{D}, \mathbf{E}, \mathbf{G}$, and $\mathbf{L}$. ${ }^{*} P<0.05,{ }^{*} P<0.01$, and ***P $<0.001$, by unpaired, 2 -tailed Student's $t$ test.

cells (Supplemental Figure 2F). Overall, these results suggest that ATMs are potential targets of NPFF.

The metabolic effects of ATMs are determined by their activation state (2). M1 activation of ATMs contributes to AT inflam- mation, which can exacerbate IR in obese AT. M1 activation can be triggered in vitro by treatment with various agents such as LPS, IFN- $\gamma$, or palmitic acid (5). IL-4 induces M2 activation, which is considered metabolically beneficial in AT, since it triggers a 
A

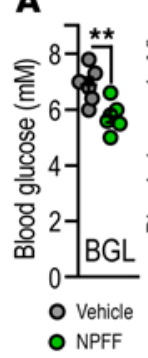

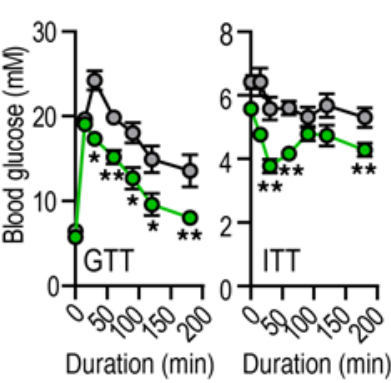

B
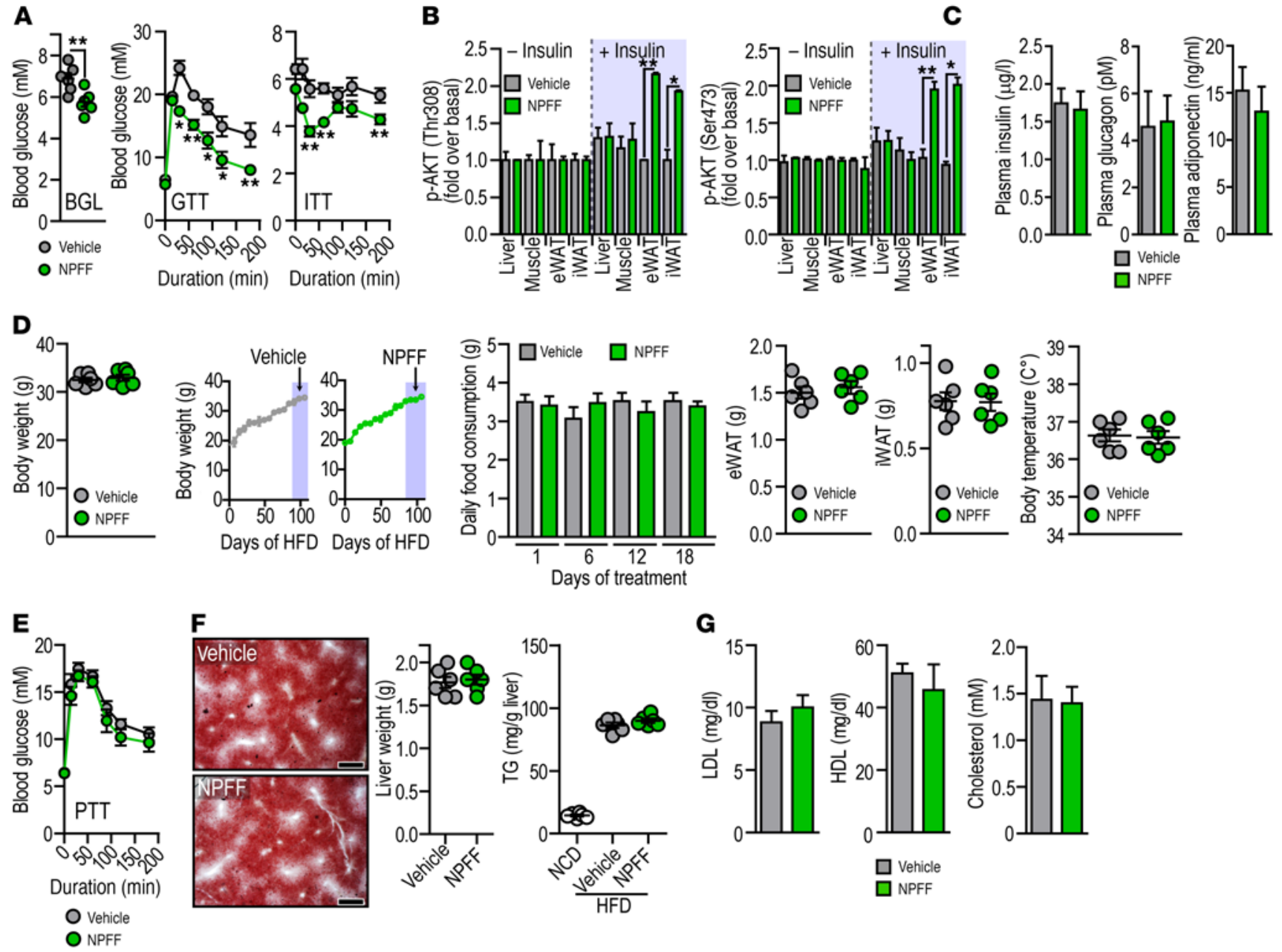

Figure 3. Metabolic phenotyping of HFD-fed mice after NPFF treatment. (A) HFD-fed mice were treated with vehicle or NPFF daily, as shown in Figure $2 \mathrm{H}$. Basal blood glucose level (BCL) after a 4-hour fast, glucose tolerance test (GTT), and insulin sensitivity test (ITT) $(n=6)$. (B) Insulin-induced AKT phosphorylation in liver, quadriceps muscle, eWAT, and iWAT. Total AKT and p-AKT (Ser308 and Thr473) levels were measured by ELISA ( $n=6)$. (C) Plasma insulin, glucagon, and adiponectin levels $(n=6)$. (D) Body weight, weight gain, food consumption, eWAT and iWAT weight, and body temperature (measured at 9:00 am) $(n=6)$. (E) PTT $(n=6)$. (F) Oil red 0 staining of liver sections. Scale bars: $100 \mu \mathrm{m}$. Liver weights and liver triglycerol (TC) content. As a comparison, NCD liver TG content is also shown $(n=6)$. (G) Plasma lipid profile $(n=6)$. ${ }^{*} P<0.05$ and ${ }^{* *} P<0.01$, by unpaired, 2-tailed Student's $t$ test and 2-way ANOVA (A) and 1-way ANOVA with Dunnett's post-hoc test (B).

pro-resolving ATM phenotype that can impede AT inflammation and IR (2). Thus, to question whether Npffr2 transcription was dependent on the ATM activation state, we treated murine ATMs with LPS or IFN- $\gamma$ and found that Npffr 2 transcription was diminished in response to either treatment (Figure 1M). Obese AT is rich in M1-activated ATMs (2). To test whether obesity-induced ATM activation modulated Npffr 2 transcription, we rendered C57BL/6 mice obese by placing them on a high-fat diet (HFD) for 12 weeks (body and AT weights are shown in Supplemental Figure 3A). When compared with normal diet-fed mice, the total ATM population from HFD-fed mice had diminished Npffr2 transcription (Figure 1M) and reduced NPFFR2 protein levels (Supplemental Figure $2 \mathrm{G}$ ), which was reflected by the reduced number of NPF$\mathrm{FR}^{+}{ }^{+}$ATMs (Figure 1N). Murine ATMs treated in vitro with IL-4 increased their expression of Npffr2 (Figure 1O), which was proportional to the duration of IL-4 exposure. Human ATMs also had upregulated NPFFR 2 transcription following IL-4 treatment (Figure 1P), and IL-13 had a similar effect (Supplemental Figure 2H).
Plasma levels of NPFF in mice and humans are sufficient for M2 activation of ATMs. Since Npffr 2 expression was upregulated by IL-4, we questioned whether NPFF impacted the IL-4-induced transcriptional changes in ATMs. Data on NPFF bioavailability and receptor binding are scarce. Thus, we first determined the effective dose of NPFF that could activate NPFFR2 signaling in ATMs. NPFF bound to murine ATMs with a $K_{D}$ of $0.37 \mathrm{nM} \pm 0.01$ nM (Figure 2A and Supplemental Figure 3B). NPFFR2 belongs to the family of $G_{i}$ protein-linked neuropeptide receptors (14), and, accordingly, NPFF treatment strongly reduced cAMP levels in ATMs, with a half-maximal effective concentration $\left(\mathrm{EC}_{50}\right)$ of 0.40 $\pm 0.01 \mathrm{nM}$ (Figure 2B and Supplemental Figure 3C).

To estimate the NPFF concentration to which ATMs can be exposed in vivo, we determined its concentration in mouse and human plasma (details of the mass spectrometric analysis and ELISA for plasma NPFF levels can be found in Supplemental Figure $4, \mathrm{~A}-\mathrm{D}$ ). The plasma levels of NPFF were $0.53 \mathrm{nM} \pm 0.02 \mathrm{nM}$ in lean 8-week-old male C57BL/6 mice and $0.48 \mathrm{nM} \pm 0.03 \mathrm{nM}$ 
A

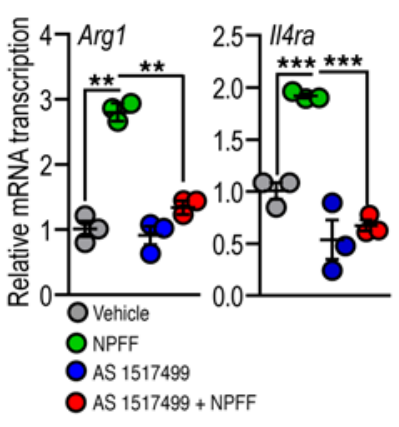

E

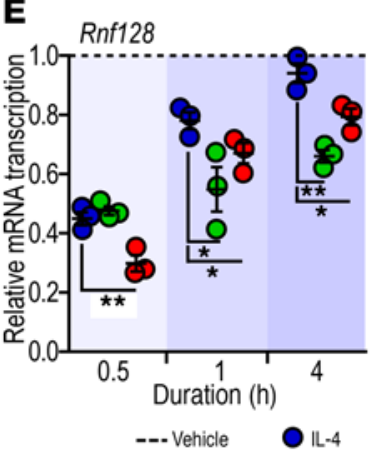

B

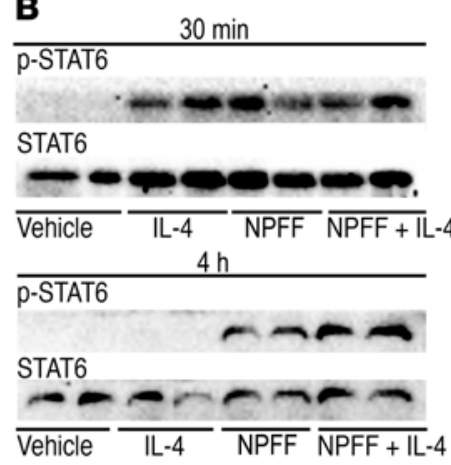

C

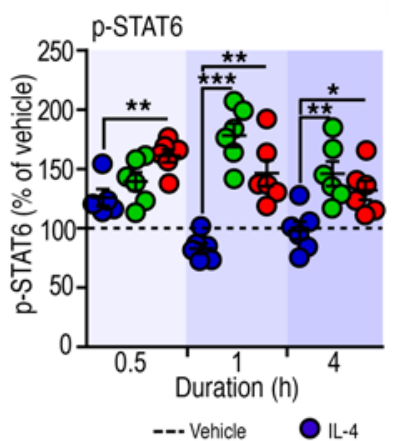

D

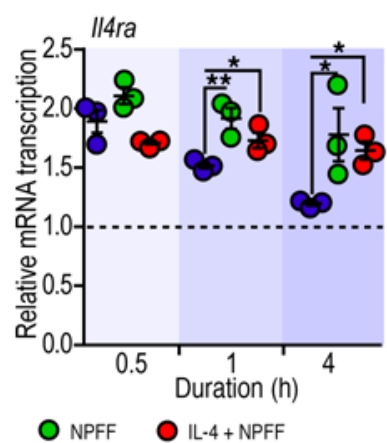

H
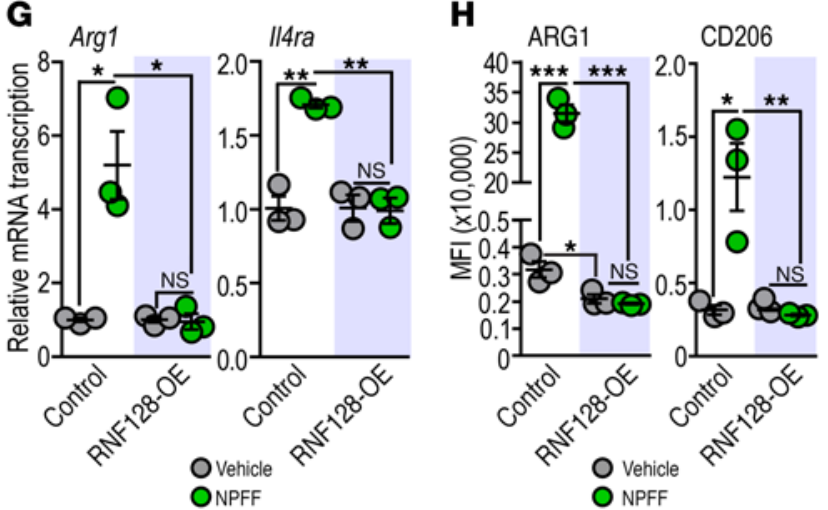
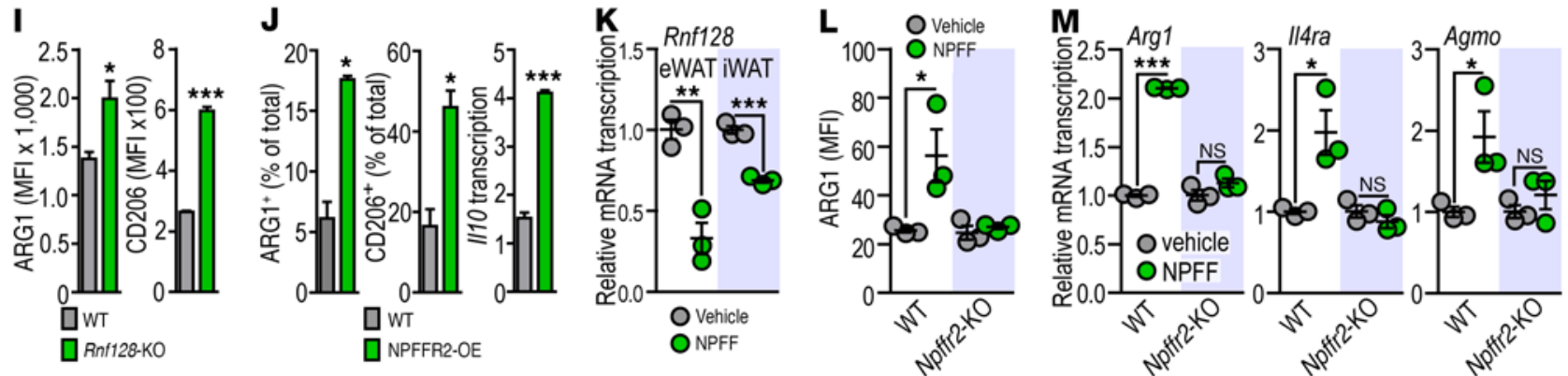

Figure 4. NPFF inhibits p-STAT6 decay and triggers M2 activation of ATMs. (A) Effect of the STAT6 inhibitor AS1517499 (200 nM) on NPFF-induced Arg1 and II4ra transcription. ATMs were treated with $0.5 \mathrm{nM} \mathrm{NPFF}$ for 1 hour. (B) Western blot of p-STAT6/STAT6 in ATMs treated with $100 \mathrm{ng} / \mathrm{ml}$ IL-4 or 0.5 nM NPFF. Each lane corresponds to pooled ATMs from 3 to 5 mice. Results are representative of 3 independent experiments. (C) p-STAT6 levels following $10 \mathrm{ng} / \mathrm{ml} \mathrm{IL-} 4$ and $0.5 \mathrm{nM}$ NPFF treatment, as measured by in-cell ELISA. (D) Effect of $10 \mathrm{ng} / \mathrm{ml} \mathrm{IL-4}$ and $0.5 \mathrm{nM}$ NPFF on the transcription of $/ / 4 \mathrm{ra}$ in ATMs. (E) Effect of $10 \mathrm{ng} / \mathrm{ml} \mathrm{IL-4}$ and $0.5 \mathrm{nM}$ NPFF on the transcription of Rnf128 in ATMs. (F) RNF128 levels in lysates of ATMs treated with $10 \mathrm{ng} / \mathrm{ml}$ IL-4, $0.5 \mathrm{nM}$ NPFF, or their combination. In C-F, the dotted lines represent the levels with vehicle treatment. (G and $\mathbf{H})$ Relative transcription and MFI of M2 markers in control and RNF128-overexpressing (RNF128-OE) J774A.1 macrophages after a 4-hour treatment with vehicle or $0.5 \mathrm{nM}$ NPFF. (I) MFI of M2 markers in ATMs from WT and Rnf128-KO mice. Data points represent pooled ATMs from 2 mice. The experiment was conducted 2 times. (J) Percentage of M2 ATMs and relative transcription of $/ 110$ in ATMs from WT and NPFFR2-overexpressing (NPFFR2-OE) mice $(n=6)$. (K) Relative transcription of Rnf128 in ATMs from vehicle-treated and NPFF-treated HFD-fed mice (treatment scheme as in Figure 2H). Data points represent pooled ATMs from 2 mice. The experiment was conducted $\mathbf{2}$ times. In $\mathbf{A}$ and $\mathbf{C}-\mathbf{F}$, the data points represent pooled ATMs from 3 mice. The experiment was conducted 3 times. ( $\mathbf{L}$ and $\mathbf{M}$ ) Arginase 1 expression (L) and transcriptional changes (M) in WT and Npffr2-KO ATMs evoked by $0.5 \mathrm{nM}$ NPFF within 1 hour. ATMs harvested from 6 mice and treated in triplicate. The experiment was conducted 6 times. ${ }^{*} P<0.05,{ }^{*} P<0.01$, and ${ }^{* * *} P<0.001$ by 1 -way ANOVA with Dunnett's post-hoc test (A and $\mathbf{C}-\mathbf{H}$ ) and unpaired, 2-tailed Student's $t$ test (I-M).

in nonobese volunteers (Figure 2C). These values fit well with the $\mathrm{EC}_{50}$ of NPFFR2 in ATMs. We found no correlation between age and sex and human plasma NPFF levels (Supplemental Figure 4E). By contrast, in HFD-fed mice and morbidly obese patients, the plasma concentrations of NPFF were below the $\mathrm{EC}_{50}$ for NPFFR2 (Figure 2C; see the Supplemental Information for a summary of the patients' metabolic parameters). This reduction in plasma NPFF levels was reversed by caloric restriction (CR) in both HFD- fed mice and morbidly obese patients (Figure 2C). We found that intermittent fasting also increased plasma NPFF levels in lean volunteers (Figure 2C). Indeed, plasma glucose levels negatively correlated with plasma NPFF levels in both mice and humans (Supplemental Figure 4F).

NPFF is released from the hypothalamus and from peripheral nerves within the adrenal gland (13). In addition, we identified the pancreas as a site of Npff expression (Supplemental Fig- 
A
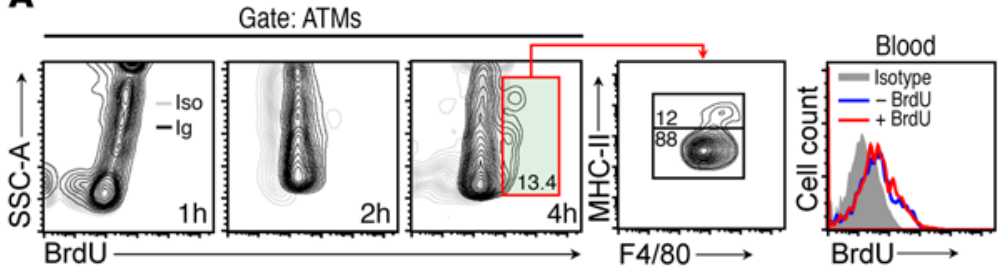

B

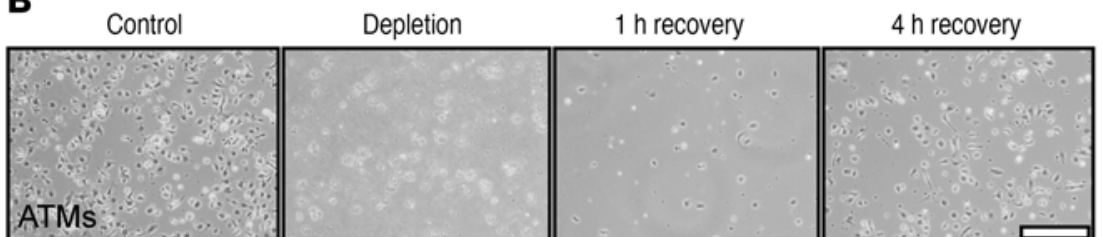

D

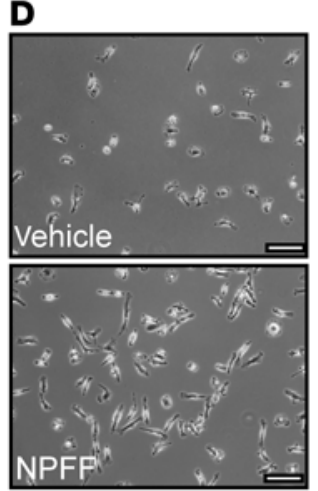

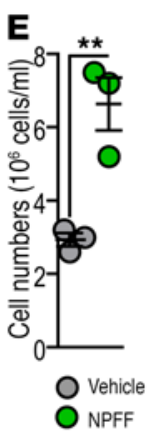

$F$

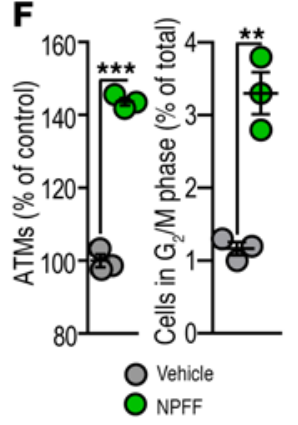

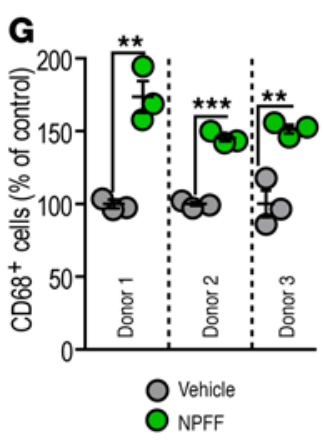

C
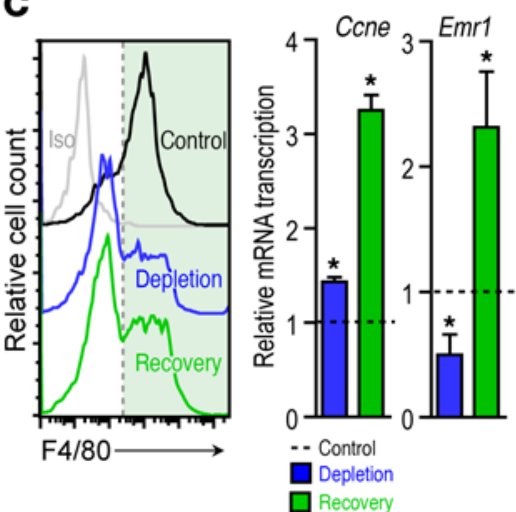

Figure 5. NPFF increases the proliferation of ATMs. (A) Mice were injected with BrdU, and ATMs were isolated after BrdU administration as shown. FACS analysis of BrdU ${ }^{+}$ATMs from eWAT. BrdU incorporation was also assessed in blood cells after 4 hours. SSC-A, side scatter area. (B) SVF cells pooled from eWAT from 6 lean mice were cultured in vitro (Control). SVF cells were incubated with clodronate liposomes for 1 hour (Depletion). Apoptotic cells were removed by washing, and surviving cells were allowed to recover in fresh medium for 1 hour and 4 hours. Scale bar: $100 \mu \mathrm{m}$. (C) FACS analysis of cells from the experiment shown in B (left). Relative transcription of Ccne and Emr1 in SVF cells treated with clodronate liposomes (blue bars) and allowed to recover for 2 hours (green bars) (right). Dashed lines indicate gene transcription level in control cells. (D) Overview of ATM cultures treated with vehicle or $0.5 \mathrm{nM}$ NPFF (18 hours). Scale bars: $50 \mu \mathrm{m}$. (E) Number of ATMs after treatment with vehicle or $0.5 \mathrm{nM}$ NPFF for 18 hours. (F) Proliferation of mouse ATMs cultured in vitro and treated for 18 hours with $0.5 \mathrm{nM}$ NPFF. ATMs were pooled from 3 to 5 mice and treated in triplicate in D-F. (G) Proliferation of human ATMs in response to treatment with $0.5 \mathrm{nM} \mathrm{NPFF}$ for 18 hours. ATMs were isolated and cultured in triplicate from each donor. (H) HFD-fed mice were treated with vehicle or NPFF as shown in Figure $2 \mathrm{H}$. Relative transcription of the macrophage markers Emr1 and Cd64 (also known as Fcgr1a) was measured in SVF. Each data point represents pooled ATMs from eWAT of 2 mice. The experiment was conducted 2 times. Quantity of ATMs in eWAT after treatment with vehicle or NPFF $(n=6)$. The values for iWAT are shown in Supplemental Figure 16C. (I) Proliferation of in vitro cultured WT and Npffr2-KO ATMs treated with $0.5 \mathrm{nM}$ NPFF for 4 hours. ATMs were pooled from 6 mice and treated in triplicate. The experiment was conducted 6 times. ${ }^{*} P<0.05$ ${ }^{* *} P<0.01$, and ${ }^{* * *} P<0.001$, by unpaired, 2-tailed Student's $t$ test.

ure 5). In both mice and humans, the majority of the pancreatic polypeptide- and somatostatin-containing endocrine cells of the islets contained NPFF-immunoreactive material (Supplemental Figure 6). Of note, pancreatic transcription of Npff was reduced in HFD-fed mice, whereas hypothalamic Npff levels remained unchanged (Supplemental Figure 6I).

We treated ATMs with $0.5 \mathrm{nM}$ NPFF, a concentration that was within the plasma level range in lean mice and humans and an effective dose for reducing intracellular cAMP levels (Figure $2, \mathrm{~A}-\mathrm{C}$ ). This concentration of NPFF increased the transcription of Npffr2 and NPFFR2 in mouse and human ATMs, respectively (Figure 2D), and mimicked the effect of IL-4 (Figure 2, E-G, and Supplemental Figure 7A).

In mouse ATMs, both NPFF $(0.5 \mathrm{nM})$ and IL-4 $(10 \mathrm{ng} / \mathrm{ml})$ increased the transcription of arginase 1 (Arg1), IL-10 (Il1O), and IL-4 receptor $\alpha$ (Il4ra), all of which are effectors of M2 macrophages
(Figure 2E). These transcriptional changes were concomitant with reduced transcription of the M1 markers IL-6 (Il6) and TNF- $\alpha$ (Tnfa) (Supplemental Figure 7A). IL-4 and NPFF also upregulated Agmo (Figure 2E), which encodes alkylglycerol monooxygenase (AGMO, EC 1.14.16.5). AGMO has antiinflammatory potential $(15,16)$, and Agmo transcription was reduced in response to LPS and IFN- $\gamma$ (Supplemental Figure 7B). In human ATMs, NPFF treatment increased the expression of CD206 (also known as MRC-1) (Figure 2F), which is associated with M2-like, pro-resolving activation of human macrophages $(17,18)$ (Supplemental Figure $7 \mathrm{C}$ ). Moreover, in human ATMs, NPFF increased the transcription of IL4RA and AGMO, the human homologs of Il4ra and Agmo (Supplemental Figure 7C). Prolonged exposure to NPFF $(24 \mathrm{~h})$ led to an increase in arginase 1 expression in mouse ATMs, which was more robust than that provoked by IL-4 (Figure 2G). Peritoneal macrophages and Kupffer cells, which have moderate or negligible NPFFR2 expression when 

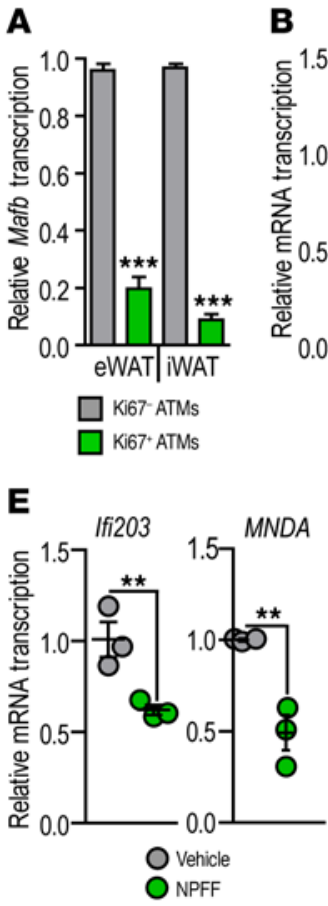
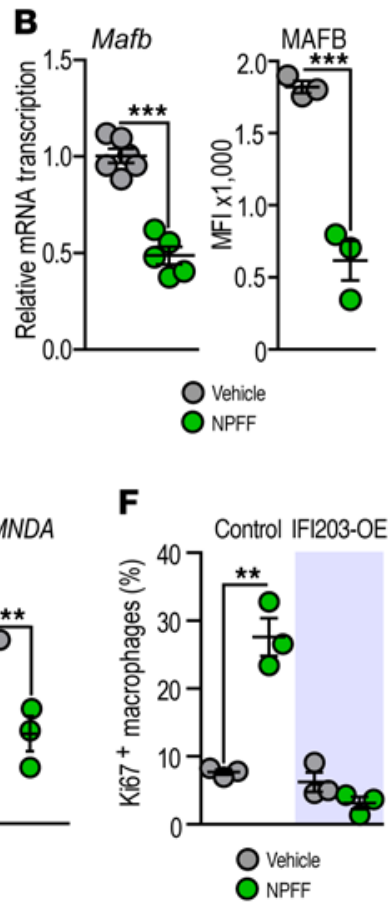
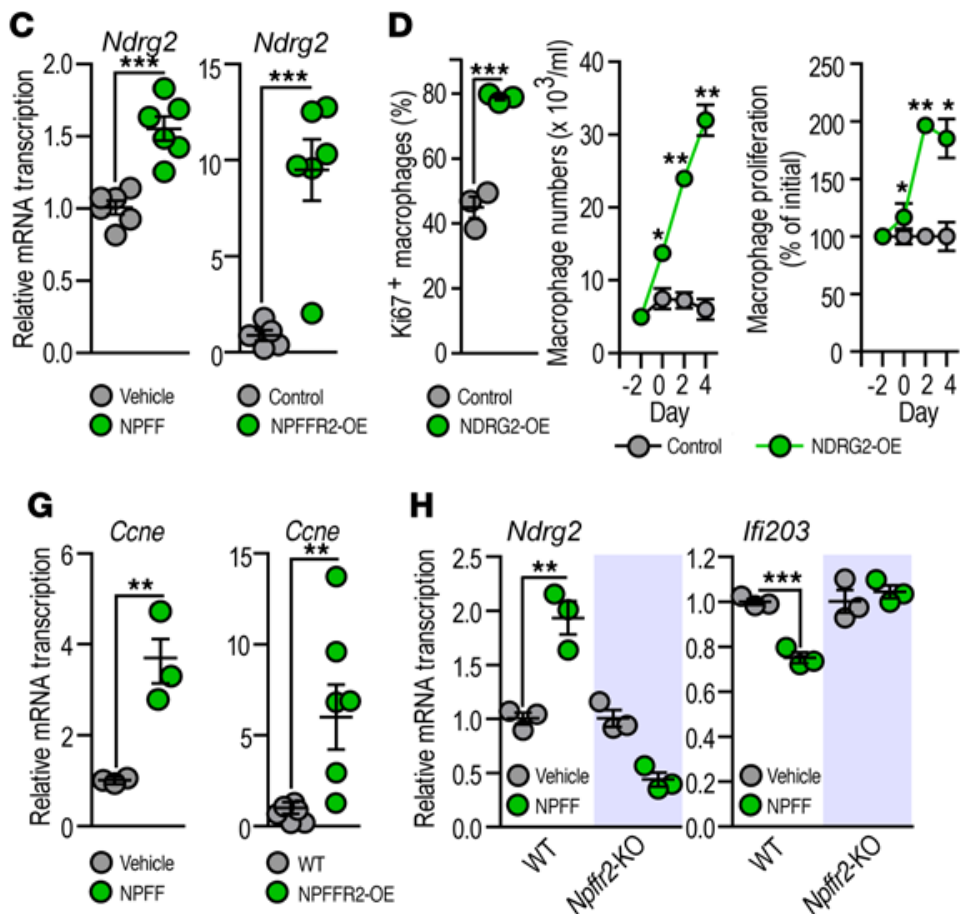

H

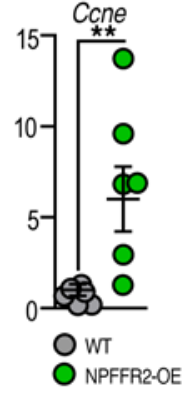

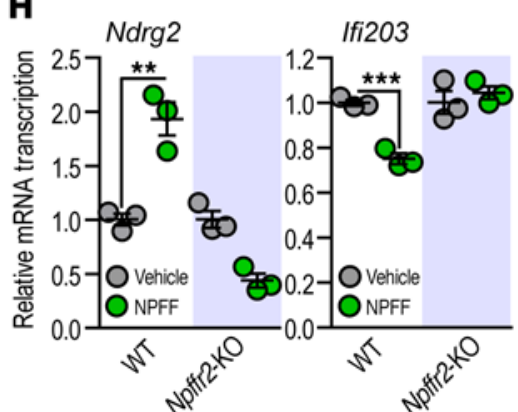

Figure 6. NPFF suppresses the cell cycle inhibiting gene transcription in ATMs. (A) Relative transcription of Mafb in Ki67- and Ki67 ${ }^{+}$ATMs $(n=3)$. (B) Relative transcription of Mafb and MFI of MAFB protein in mouse ATMs treated with vehicle or $0.5 \mathrm{nM}$ NPFF for 4 hours ( $n=5$ and 3 , respectively). (C) Relative transcription of Ndrg2 in ATMs treated with $0.5 \mathrm{nM} \mathrm{NPFF}$ for 0.5 hours $(n=5)$ and in freshly isolated ATMs from WT or NPFFR2-overexpressing (NPFFR2-OE) mice $(n=6)$. (D) Cell numbers and proliferation of control and NDRG2-overexpressing (NDCR2-0E) U937 macrophages ( $n=3$ ). Quantity of Ki67 ${ }^{+}$cells in control and NDRG2-overexpressing U937 macrophage cultures after 4 days of culture $(n=3)$. (E) Relative transcription of Ifi203 in mouse ATMs in response to treatment for 0.5 hours with $0.5 \mathrm{nM}$ NPFF. Relative transcription of MNDA in human ATMs in response to a 4-hour treatment with $0.5 \mathrm{nM} \mathrm{NPFF}(n=3)$. (F) Effect of a 4-hour treatment with 0.5 nM NPFF on the proliferation of control and IFI203-overexpressing (IFI203-0E) J774A.1 macrophages $(n=3)$. (G) Relative transcription of Ccne in ATMs treated with $0.5 \mathrm{nM}$ NPFF for 4 hours and in WT and NPFFR2-overexpressing ATMs $(n=3$ and 7, respectively). (H) Effect of $0.5 \mathrm{nM} \mathrm{NPFF}$ on Ndrg2 and IfizO3 transcription in WT and Npffr2-KO ATMs. ATMs were pooled from 3 mice and treated in triplicate. The experiment was conducted 3 times. ${ }^{*} P<0.05$, ${ }^{* *} P<0.01$, and ${ }^{* * *} P<0.001$, by unpaired, 2 -tailed Student's $t$ test.

compared with ATMs (Figure 1L and Supplemental Figure 2C), failed to upregulate Arg1, Il4ra, or Agmo or reduce Tnfa following 0.5 nM NPFF treatment in vitro (Supplemental Figure 7D). To evaluate whether the plasma levels of NPFF found in obesity could effect changes in ATMs, we treated mouse ATMs with $0.2 \mathrm{nM} \mathrm{NPFF}$; however, this concentration failed to induce M2 activation (Supplemental Figure 7E), corroborating the notion that plasma levels of NPFF in obesity are insufficient to affect ATM activation.

NPFF causes M2 activation of ATMs in vivo. Our finding that diet-induced obesity reduced NPFF plasma levels and diminished the prevalence of Npffr2-expressing ATMs suggested that the M2-activating effect of NPFF is lost in this setting. This prompted us to explore whether NPFF replacement could induce M2 activation and yield metabolic benefits in diet-induced obesity. Accordingly, HFD-fed mice were treated with $4 \mathrm{nmol} / \mathrm{kg} /$ day NPFF for 18 days (Figure 2H), which increased plasma levels of NPFF to $0.494 \mathrm{nM}$ $\pm 0.074 \mathrm{nM}$ versus $0.109 \mathrm{nM} \pm 0.019 \mathrm{nM}$ in vehicle-treated mice at the end of the study (Supplemental Figure 8A) and was within the range observed in lean mice (Figure 1C). We analyzed ATMs in both eWAT and iWAT, since AT depots can have a specific impact on metabolism and some hormones or drugs can have adipose depot-specific effects $(1,2)$. The inflammatory state was comparable between the 2 adipose depots after HFD feeding (Supplemental Figure 7F and Supplemental Figure 8, B-D). The level of transcrip- tion of Npffr2, Arg1, Il10, Il4ra, Agmo, Cd163, and Cd206 was higher in ATMs of both depots from NPFF-treated mice than in ATMs from vehicle-treated mice, with a concomitant reduction of Il6, Tnfa, and Nos 2 transcription (Figure 2, I-K, and Supplemental Figure 8, B-D). These transcriptional changes are characteristic of diminished AT inflammation and have been shown to be protective against the development of IR $(19,20)$. Obese AT accumulates CD11 $\mathrm{c}^{+} \mathrm{ATMs}$, which are associated with the dominance of M1-ATM activation (20). We found that NPFF treatment reduced Cd11c (also known as Itgax) transcription and the amount of CD11 $\mathrm{c}^{+} \mathrm{ATMs}$ in eWAT and iWAT (Supplemental Figure 7F). Indeed, glucose tolerance as well as AT insulin sensitivity were increased after NPFF treatment (Figure 3, A and B), whereas plasma insulin, glucagon, and adiponectin levels (Figure 3C), as well as body weight, food intake, AT weight, and body temperature (Figure 3D) were unaffected by NPFF treatment. Moreover, gluconeogenesis measured by a pyruvate tolerance test (PTT) (Figure 3E), the degree of change in fatty liver (Figure 3F), and the plasma lipid profile (Figure 3G) were unaffected by NPFF. NPFF treatment reduced AT inflammation (Supplemental Figure 7G), however, liver and muscle inflammatory states were unchanged, and NPFF had negligible effects on the transcription of Il6, Tnfa, Nos2, and Arg1 in Kupffer cells and skeletal muscle (Supplemental Figure 7H), which is in striking contrast to its effect on ATMs in vivo (Figure 2, I-K). These findings suggest that 

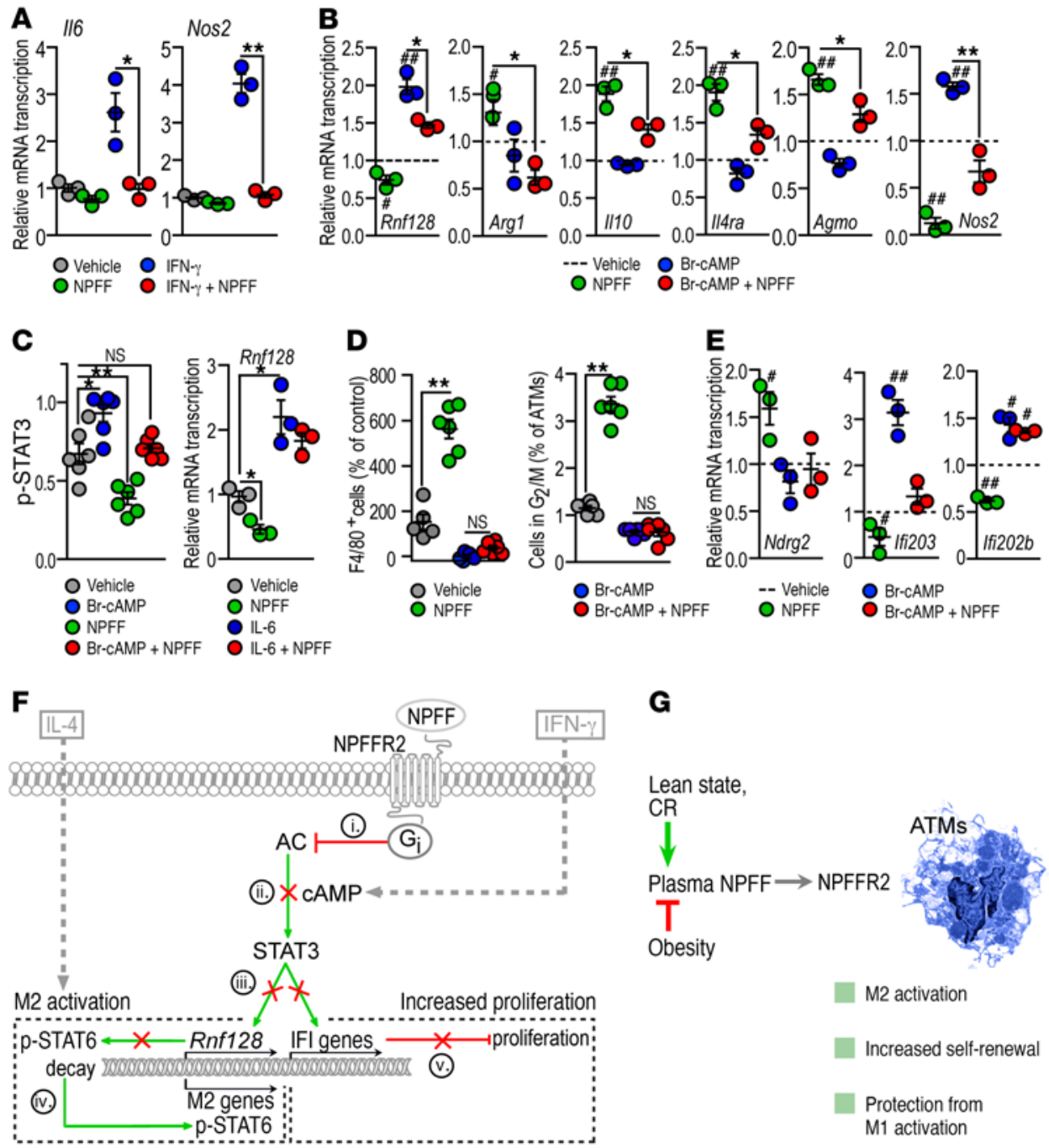

Figure 7. NPFF/NPFFR2 inhibits IFN signaling. (A) Effect of a 4-hour treatment with $0.5 \mathrm{nM} \mathrm{NPFF}, 2.5 \mathrm{ng} /$ $\mathrm{ml}$ IFN- $\gamma$, or their combination on $/ 16$ and Nos2 transcription in mouse ATMs. (B) Effect of $500 \mu \mathrm{M} \mathrm{Br}$-cAMP on the transcriptional changes in mouse ATMs evoked within 0.5 hours by treatment with 0.5 nM NPFF. (C) STAT3 phosphorylation within 20 minutes in mouse ATMs following treatment with $0.5 \mathrm{nM}$ NPFF and 500 $\mu \mathrm{M} \mathrm{Br}$-CAMP (left). Effect of a 1-hour treatment with $0.5 \mathrm{nM} \mathrm{NPFF}$ and $100 \mathrm{ng} / \mathrm{ml} \mathrm{IL-6}$ on Rnf128 transcription in mouse ATMs. The effect of NPFF was also tested following a 20-minute preincubation with IL-6 (right). (D) Proliferation of ATMs treated for 18 hours with $0.5 \mathrm{nM}$ NPFF, $500 \mu \mathrm{M} \mathrm{Br}$-cAMP, or their combination. (E) Effect of $500 \mu \mathrm{M} \mathrm{Br}$-cAMP and $0.5 \mathrm{nM}$ NPFF on Ndrg2 and Ifizo3 transcription in ATMs and Ifizo2b transcription in J774A.1 macrophages. In A-E, each data point represents pooled ATMs from 3 to 5 mice. In E, triplicates of j774A.1 cultures were used. ${ }^{*} P<0.05$ and ${ }^{* *} P<0.01$ for the indicated comparisons and ${ }^{\#} P<0.05$ and ${ }^{\# \#} P<0.01$ versus vehicle control; 1-way ANOVA with Dunnett's post-hoc test. (F) NPFF action in macrophages (simplified scheme): (i) NPFF/NPFFR2 blocks adenylate cyclase (AC); (ii) NPFF reduces CAMP levels; (iii) STAT3 signaling is inhibited; (iv) Reduced Rnf128 transcription delays p-STAT6 decay; and (v) Inhibition of IFN-inducible (IFI) genes. (C) Role of NPFF/NPFFR2 signaling in ATMs. Morbid obesity reduces plasma NPFF to levels below the effective dose of NPFF in ATMs.

ATMs are the major targets of NPFF. To further confirm the direct antiinflammatory effect of NPFF on ATMs, we treated them in vitro with palmitic acid to mimic M1 activation caused by lipid overload. We observed that NPFF exposure increased Il10 transcription and abolished Il6 and Tnfa transcription in palmitic acid-treated ATMs (Figure 2L). NPFF treatment also inhibited nuclear translocation of NF- $\mathrm{KB}$ in an IL-10-dependent manner (Supplemental Figure 9).

NPFF inhibits phosphorylated STAT6 decay in ATMs. It is known that IL-4 exerts its effects on macrophage activation via STAT6 sig- naling (21). We found that the effect of NPFF on macrophage activation was also dependentonSTAT6, as shown by the reduction of Arg1 and II4ra expression in NPFF-treated ATMs cotreated with the STAT6 inhibitor AS1517499 (Figure 4A; further details on the inhibitory effect of AS1517499 on STAT6 signaling are provided in Supplemental Figure 10A). Accordingly, ATMs cotreated with AS1517499 lost their capacity to phosphorylate STAT6 following NPFF treatment (Supplemental Figure 10B). The level of phosphorylated STAT6 (p-STAT6) was sustained by NPFF for a longer period than was observed with IL-4 (Figure $4, \mathrm{~B}$ and $\mathrm{C}$ ), and, as a consequence, transcription of the STAT6 target Il4ra remained elevated for an extended period (Figure 4D). This result is consistent with the finding that arginase 1 expression was higher in NPFFtreated ATMs after 24 hours than in IL-4-treated ATMs (Figure 2G).

These findings led us to investigate whether NPFF inhibited the inactivation of STAT6 signaling. While little is known about the mechanisms that inactivate IL-4/STAT6 signaling in macrophages, proteasomal degradation of p-STAT6 is thought to be an important switch-off mechanism (22). It has been recently demonstrated that the E3 ubiquitinprotein ligase RNF128 (EC 6.3.2.), also known as GRAIL, is responsible for p-STAT6 ubiquitination and proteasomal degradation in Th2 cells $(23,24)$. The effect of NPFF on transcription of the STAT6 target Il4ra was similar to that of RNF128 deficiency in Th2 cells, which increases the transcription of Il4ra (24). This prompted us to postulate that the reduced level of RNF128 was responsible for the effect of NPFF in ATMs. Therefore, we measured Rnf128 transcription after IL-4 and NPFF treatment in ATMs. IL-4 treatment reduced Rnf128 transcription within 30 minutes, but this effect was lost at longer (1- or 4-h) treatment durations (Figure 4E). Protein expression of RNF128 was strongly decreased by IL-4 within 1 hour; however, the initial level of RNF128 was restored in ATMs treated for 10 hours with IL-4 (Figure 4F). By contrast, in NPFF-treated ATMs, restoration of Rnf128 mRNA and RNF128 protein levels did not occur if the ATMs were treated with NPFF (Figure 4, E and F). 
The above findings suggest that reduced RNF128 is responsible for the maintained STAT6 phosphorylation observed in NPFF-treated ATMs. Supporting this possibility, NPFF failed to maintain STAT6 phosphorylation in J774A.1 macrophages overexpressing RNF128 (Supplemental Figure 10C) and did not induce M2 activation (Figure 4, G and $\mathrm{H}$ ). In turn, ATMs from RNF128-deficient mice showed increased p-STAT6 levels (Supplemental Figure 11A) and expression of arginase 1 and CD206, both of which are hallmarks of M2 activation (Figure 4I and Supplemental Figure 11B; details on the generation and phenotype of RNF128-deficient mice are provided in Supplemental Figure 11, C-E). Similar to RNF128 deficiency, ATMs overexpressing NPFFR2 had increased expression of arginase 1 and CD206 and transcription of Il1O (Figure 4J, and Supplemental Figure 12A; details on the generation and phenotype of NFPFFR2-overexpressing mice are provided in Supplemental Figure 12, B-E). When HFDfed mice were treated with NPFF (Figure $2 \mathrm{H}$ ), the transcription of Rnf128 was reduced in ATMs (Figure 4K). Human ATMs also had reduced $R N F 128$ transcription following treatment with $0.5 \mathrm{nM}$ NPFF in vitro (Supplemental Figure 7C).

The M2-activating effects of NPFF were mediated by NPFFR2, since ablation of NPFFR2 abolished NPFF-induced p-STAT6 (Supplemental Figure 10D) and NPFF-induced transcriptional changes (Figure 4, L and M, and Supplemental Figure 8E; details on the generation and phenotype of NPFFR2-deficient mice are provided in Supplemental Figure 13, A-F). Similarly, pharmacological inhibition of NPFFR2 abolished NPFF-induced transcriptional changes (Supplemental Figure 14, A and B).

NPFF increases ATM self-renewal. It has been reported that ATMs can proliferate (4), and recent studies suggest that IL-4 promotes tissue-resident macrophage proliferation $(25,26)$. Nevertheless, increased ATM proliferation contributes to the accumulation of M1-ATMs in obese AT $(1,4)$; however, signals controlling selfrenewal of ATMs are poorly understood (1). Whether local proliferation is relevant for ATM replenishment in lean AT is also unknown. Nonetheless, this possibility was supported by our findings that $\mathrm{Ki}^{+} 7^{+}$ATMs were present in both eWAT and iWAT (Supplemental Figure 15), and BrdU was incorporated into ATMs (Figure 5A). The time window for BrdU incorporation into ATMs matched the ATM proliferation rate reported for obese AT in mice (27). BrdU was not incorporated into blood monocytes (Figure 5A). Altogether, these findings suggest that a proliferating ATM population exists in lean AT, which replenishes ATMs. To study this possibility in more detail, isolated ATMs were maintained in vitro and challenged with clodronate liposomes. Under this condition, blood monocytes cannot be recruited, and new ATMs should be generated from a bone marrow-independent stem cell pool. Shortly after removal of the clodronate liposomes, we found that newly formed ATMs were present in the culture (Figure $5 \mathrm{~B}$ ), as demonstrated by the increased number of $\mathrm{F} 4 / 80^{+}$cells and increased $\mathrm{Emr} 1$ (encoding F4/80 antigen) transcription (Figure 5C). ATM recovery was associated with increased cyclin E (Ccne) transcription (Figure 5C). We observed that replenishment of ATMs was also increased in vitro by NPFF treatment (Figure 5, D and E).

To corroborate the finding that NPFF increases ATM selfrenewal, we treated mouse and human ATMs in vitro with 0.5 nM NPFF. The number and proliferation of both mouse and human ATMs were increased by NPFF treatment (Figure 5, F and $G$ ). Similarly, the number of ATMs was increased following NPFF treatment of mice (Figure 5H, Supplemental Figure 14C, and Supplemental Figure 16G). ATMs lacking NPFFR2 expression failed to upregulate proliferation in response to NPFF (Figure 5I), and pharmacological inhibition of NPFFR2 abolished the effect of NPFF on macrophage proliferation (Supplemental Figure 14C and Supplemental Figure 16, A-F). Accordingly, NPFFR2overexpressing mice had increased ATM numbers and proliferation (Supplemental Figure 12F), while NPFFR2-deficient mice had reduced ATM numbers and proliferation (Supplemental Figure 13, $\mathrm{G}$ and $\mathrm{H}$ ), without an effect on peripheral blood hematology (Supplemental Figure 12G and Supplemental Figure 13I).

Proliferating ATMs in both eWAT and iWAT had lower expression levels of the transcription factor MAF bZIP transcription factor B (Mafb) than did quiescent ATMs (Figure 6A). Reduced Mafb transcription allows macrophage proliferation $(25,28,29)$, and we have previously demonstrated the impact of low Mafb transcription levels on the proliferation of macrophage precursors (28). When mouse ATMs were treated with NPFF, mRNA and protein expression levels of $M a f b$ were decreased (Figure 6B).

NPFF treatment or NPFFR2 overexpression increased the transcription of $\mathrm{N}-\mathrm{Myc}$ downstream-regulated gene 2 ( Ndrg2) in ATMs (Figure 6C). NDRG2 can increase cell proliferation in specific cell types (30); however, its impact on macrophage proliferation is not known. Overexpression of NDRG2 in U937 macrophages (Supplemental Figure 13J) led to increased proliferation, mimicking the effect of NPFF (Figure 6D). Furthermore, NPFF treatment decreased the transcription of IFN-inducible 200 (IFI200) family members, which inhibit proliferation and suppress growth in various cell types including macrophage progenitors (31). Ifi203 was the relevant IFI200 family member expressed in C57BL/6-derived ATMs, whereas Ifi202b, Ifi204, and Ifi2O5 were expressed in BALB/c-derived J774A.1 macrophages (Figure $6 \mathrm{E}$ and Supplemental Figure 17A), in agreement with a previous study on strain-specific expression of Ifi200 genes (32). We found that overexpression of IFI203 (Supplemental Figure 13K) abrogated the effect of NPFF on macrophage proliferation (Figure $6 \mathrm{~F})$. Moreover, in human ATMs, myeloid cell nuclear differentiation antigen (MNDA), a homolog of the murine IFI200 family, was suppressed by NPFF (Figure 6E).

NPFF-treated ATMs and ATMs overexpressing NPFFR2 had increased Ccne transcription, further confirming the notion that NPFF/NPFFR2 signaling increases ATM proliferation (Figure 6G). The absence of NPFFR2 abolished the effect of NPFF on Ndrg2 and Ifi203 transcription in ATMs (Figure 6H). Pharmacological inhibition of NPFFR2 had a similar effect (Supplemental Figure 16F).

NPFF inhibits IFN- $\gamma$ signaling, which is key for ATM activation and proliferation. IFN- $\gamma$ treatment reduced Npffr2 transcription in ATMs (Figure 1M), and, in turn, NPFF treatment abolished the effect of IFN- $\gamma$ on the transcription of Il6 and Nos2 (Figure 7A). We found that NPFF also inhibited IFN-inducible gene transcription in J774A.1 macrophages (Supplemental Figure 17, B and C). The NPFF-regulated genes ring finger protein 128 (Rnf128), $N d r g 2$, and Ifi200 family members are IFN-responsive genes, and IFN- $\gamma$ had an effect opposite that of NPFF on their transcription (Supplemental Figure 17C). 
NPFF triggers a strong reduction of intracellular cAMP, contrasting with the effect of IFN- $\gamma$, which increases cAMP levels (3335). Thus, we hypothesized that the antagonistic effects of IFN- $\gamma$ and NPFF on CAMP synthesis were key for NPFF-induced M2 activation and proliferation of ATMs. As with IFN- $\gamma$, the cAMP analog Br-cAMP provoked a pronounced increase in $R n f 128$ transcription and thus had an effect opposite that of NPFF (Figure 7B). Br-cAMP abolished the inhibitory effect of NPFF on the transcription of Rnf128 and impeded M2 activation provoked by NPFF (Figure 7B). A recent study has shown that cAMP is involved in STAT3 signaling (35), and a STAT3 response element is present in the Rnf128 promoter (Supplemental Figure 17D). We found that Br-cAMP increased STAT3 phosphorylation in ATMs, and this effect was antagonized by NPFF (Figure 7C). When STAT3 was phosphorylated by IL- 6 before treatment, NPFF failed to reduce Rnf128 transcription (Figure 7C).

Like M2-activation, increased ATM proliferation was dependent on the reduction of intracellular cAMP levels (Figure 7D). Br-cAMP abolished the effect of NPFF on the proliferation of ATMs (Figure 7D). Further, Br-cAMP abolished the inhibitory effect of NPFF on the transcription of IFI200 family members and abrogated the NPFF-induced upregulation of Ndrg2 transcription (Figure 7E).

\section{Discussion}

This study shows that NPFF is a potent regulator of ATM quantity and quality. We demonstrate here that NPFF/NPFFR2 signaling in macrophages augments STAT6 signaling, which mimics M2 activation and enhances macrophage self-renewal. NPFF/NPFFR2 signaling is thus a common hub for the control of macrophage activation and proliferation.

It has been known for more than 20 years that human plasma contains levels of NPFF one order of magnitude higher than the levels found in cerebrospinal fluid, and, consequently, it has been hypothesized that NPFF is distributed in the bloodstream and acts as a hormone $(13,36)$. This possibility, however, has not been systematically investigated. Moreover, the known effects of NPFF on metabolism, such as appetite control and thermogenesis, were traditionally associated with its neurotransmitter function in the nervous system (7). We show for the first time to our knowledge that the peripheral effects of NPFF are relevant.

Discrepancies exist between the reported plasma and cerebrospinal fluid levels of NPFF in humans and experimentally used concentrations of $\operatorname{NPFF}(8,9,13,36-38)$, making it challenging to distinguish physiological and pharmacological effects of NPFF and to define signaling pathways activated by this neuropeptide. For instance, the inhibitory effect of micromolar amounts of NPFF on NO synthesis in macrophages has been interpreted as an antiinflammatory potential of NPFF (38). However, the NPFF concentration used was orders of magnitude higher than its physiological level, making it conceivable that toxic or nonspecific effects of high-dose NPFF occurred. Our findings show that mouse and human plasma NPFF levels match the $\mathrm{EC}_{50}$ for NPFFR2 determined in our study and by others (39) and that this concentration is effective for inducing ATM proliferation and M2 activation, with concomitant protection from M1 activation. Thus, it is conceivable that plasma NPFF has the potential to control the content and activation state of ATMs.
We show that plasma NPFF levels are reduced in human and murine obesity, together with decreased numbers of Npffr2expressing ATMs in obese AT in mice. Thus, obesity can lead to the loss of the effect of NPFF on ATMs, which is consistent with the current paradigm for the relationship between obesity and dysregulated ATM proliferation and activation (1). NPFF is known as an anorexigenic factor in rodents that acts at hypothalamic nuclei $(6,7)$. The balance between anorexigenic and orexigenic signals in the hypothalamic center of appetite control is key in obesity development (40). A reduction in plasma NPFF levels may reflect impaired NPFF release from the hypothalamic nuclei in obesity. In addition, diminished Npff transcription in the endocrine pancreas can also account for the reduced plasma NPFF pool in obesity.

As shown here, NPFF shifts macrophages to an M2 activation state. IL-4 increases NPFFR2 expression, clearly demonstrating the role of NPFF signaling in M2 macrophages. In HFD-fed mice, NPFF treatment increased Npffr 2 transcription in ATMs and triggered M2 activation of ATMs, and this effect was associated with improved glucose tolerance. Since ATMs are prominent NPFFR2expressing macrophages, there is the potential for NPFF to reduce AT inflammation. NPFFR2 expression was also detectable at lower levels in other macrophage types, such as Kupffer cells, and NPFF exerted a mild antiinflammatory effect on Kupffer cells. This, however, would augment the beneficial effect of NPFF and not challenge the role of NPFF in M2 activation of ATMs.

The mechanistic basis of NPFF action in macrophages involves diminished cAMP synthesis, which suppresses the transcription of genes involved in the IFN response (Figure 7F). NPFF suppresses the expression of the IFN-responsive gene Rnf128. Reduced Rnf128 transcription delays p-STAT6 decay in NPFF-treated macrophages, leading to the transcription of $\mathrm{M} 2$ activation genes such as Arg1, Il1O, Il4ra, and Agmo. Furthermore, NPFF increases the transcription of $N d r g 2$, a known inhibitor of STAT3 signaling (41). In addition to the augmented IL-4 signaling, NPFF also increases Il1O transcription. It is well established that IL-10 suppresses the expression of proinflammatory cytokines as well as antigen-presenting and costimulatory molecules in macrophages (42).

NPFF also suppresses the transcription of members of the IFI200 family. The IFI200 family includes proteins that accumulate in the nucleus during the IFN response and interact with various transcription factors $(32,43)$. They control the cell cycle in a wide range of cell types including macrophage progenitors $(44,45)$. IFNs cause growth arrest, and IFI200 family members are involved in this effect $(32,44)$. While it is known that the Ifi200 gene family inhibits immune cell proliferation and affects inflammation (32, 44), little is known about how IFI200 expression is controlled. Our study raises the possibility that NPFF exerts hormonal control of IFI200 transcription. This has relevance in various disease conditions such as autoimmunity, in which macrophages, IFN response, and IFI200 family members play key roles (32).

NPFF exerts its effects through NPFFR2 both in vitro and in vivo. We show that NPFF/NPFFR2 signaling is a common hub for M2 activation and increased macrophage proliferation (Figure 7F), thus enriching AT with M2-activated ATMs and impeding AT inflammation (Figure $7 G$ ). The impact of macrophage selfrenewal has been recently recognized $(4,25,27)$; however, the underlying mechanisms that are decisive for ATM proliferation 
remain enigmatic. Our findings identify an endogenous hormonal signal mechanism that impacts on ATM self-renewal.

In conclusion, this study shows that NPFF controls ATM self-renewal and M2 activation. NPFF has the unique ability to increase ATM numbers and maintain the newly formed ATMs in an M2-activated state. NPFF can also protect against AT inflammation, the main predisposing factor for IR. Thus, NPFF may have utility as a tool in the future treatment of obesity-associated dysregulation of ATM activation and proliferation.

\section{Methods}

Animals. We used C57BL/6 male mice (Charles River Laboratories), NPFFR2-deficient mice, NPFFR2-overexpressing mice, and RNF128-deficient mice on a C57BL/6 background. All mice were 8 weeks of age at the beginning of the experiments. The generation of transgenic mouse lines is described in the Supplemental Methods. Male mice were rendered obese on a 12 -week-long HFD consisting of $45 \%$ of calories from fat (RD formula; SSNIFF). The control group was fed a normal chow diet (NCD) for the same period. For CR experiments, the test group received a low-calorie diet (SSNIFF) for 2 weeks. For NPFF replacement, HFD-fed mice were injected daily with $4 \mathrm{nmol} / \mathrm{kg} \mathrm{NPFF}$ (Phoenix Pharmaceuticals Inc.) for 18 days. Each experimental group was established from 6 control and 6 treated mice.

Human samples. Plasma and fat samples were obtained between June 2015 and January 2016 at the Hospital Clínico Universitario de València. The metabolic parameters for patients and volunteers are summarized in Supplemental Table 3. Plasma samples were obtained from healthy volunteers $(n=32)$, morbidly obese patients $(n=18)$, and patients with type 1 diabetes mellitus $(n=6)$. Patient selection was based on the NIH's recommendation of a BMI above $40 \mathrm{~kg} / \mathrm{m}^{2}$. The nonobese volunteers had a BMI of 20 to $25 \mathrm{~kg} / \mathrm{m}^{2}$. To test the effect of intermittent fasting, we recruited 10 nonobese volunteers, 5 of whom followed a regime of daily 16-hour fasting for 28 days, while the other 5 volunteers did not fast. Blood samples were collected in EDTA-coated hemotubes and centrifuged, and the plasma samples were stored at $-80^{\circ} \mathrm{C}$ until further analysis. Blood glucose, insulin, and NPFF levels were assayed using commercial ELISA kits from Sigma-Aldrich and Cloud-Clone Corporation. AT samples were collected from the omental fat depot of morbidly obese patients $(n=16)$ during routine bariatric surgical intervention at the Hospital Clínico Universitario de València between June 2015 and January 2016. Eight patients followed a CR diet for 1 month before blood sampling and surgery. These patients were allowed to drink a specially formulated balanced energy drink (Vegenat SA) and other calorie-free drinks such as water, tea, coffee, and nonfat broth. Omental fat from 3 nondiabetic obese donors without CR was used for isolation and in vitro culturing of ATMs. Pancreas samples were collected from deceased organ donors (Network of Pancreatic Organ Donors, University of Florida, Gainesville, Florida, USA), fixed in paraformaldehyde (PFA), and processed for histological and gene expression analysis. Samples from 2 nondiabetic donors were used for histology. Gene expression analysis was performed for 18 control donors with no autoantibodies and no diabetes and 20 donors with type 1 diabetes mellitus (for details, see the Supplemental Information). One patient underwent radical pancreas resection, and plasma samples were collected prior to and after surgery.

Assessment of plasma NPPF levels. Plasma NPFF levels in human and murine plasma were assayed using commercial ELISA kits
(Cloud-Clone Corp.). Details of the mass spectrometric data on plasma NPFF levels and quality control for the ELISA are provided in Supplemental Figure 3.

Isolation and in vitro culture of ATMs. ATMs were isolated from eWAT and iWAT depots from mice and omental fat from human donors using collagenase digestion as described previously (3). ATMs were suspended in selection medium consisting of $2 \mathrm{mM}$ EDTA and $0.5 \%$ BSA in PBS, pH 7.4, and used for flow cytometry, transmission electron microscopy (TEM), preparative cell sorting, and RNA extraction, or were cultured in vitro (Supplemental Figure 1). For in vitro culturing, $\mathrm{CD} 11 \mathrm{~b}^{+} \mathrm{F} 4 / 80^{+}$mouse ATMs or $\mathrm{CD} 14^{+} \mathrm{CD} 68^{+}$human ATMs were retrieved with preparative cell sorting as described below. ATMs were allowed to adhere to 6-well plates in RPMI 1640 medium with 10\% FCS and were treated with NPFF (Tocris Bioscience; Phoenix Pharmaceuticals Inc.), RF9 (14), $100 \mathrm{ng} / \mathrm{ml}$ LPS from E. coli, 10

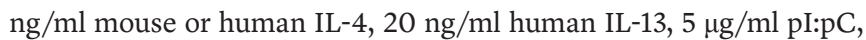
$2.5 \mathrm{ng} / \mathrm{ml} \mathrm{IFN}-\gamma, 500 \mu \mathrm{M}$ Br-cAMP (all from Sigma-Aldrich), and 100 ng/ml IL-6 (ImmunoTools) or 200 nM AS 1517499 (Axon Chemicals). NPFF purity was controlled by HPLC.

FACS analysis and preparative cell sorting. Cell density was adjusted to $10^{6}$ cells $/ \mathrm{ml}$, and analysis was performed on a BD LSR II Cell Cytometer (BD Bioscience). The antibodies used are listed in Supplemental Table 1. After a 2-hour incubation, the cells were washed in selection buffer and used for analysis. For preparative

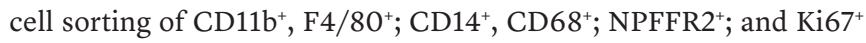
or Ki67- ATMs, we used the Aria Cell Sorter. CD11b, F4/80, CD14, CD68, and NPFFR2 staining was performed on live cells. The sorted cells were collected for Western blotting, RNA extraction, or for further TEM analysis. Ki67 staining was carried out on cells fixed with $2 \%$ PFA and permeabilized with $0.05 \%$ Triton X-100 to ensure recognition of the nuclear antigen Ki67 by the antibody. In this case, CD11b and F4/80 staining was performed on fixed cells. Sorted Ki67 ${ }^{-}$and $\mathrm{Ki}^{-} 7^{+}$ATMs were used for RNA extraction. For cell-cycle analysis, the in vitro cultured ATMs were fixed with 2\% PFA. Cells were washed with selection buffer and treated with RNAase, and the DNA was stained with propidium iodide (Sigma-Aldrich).

RNA extraction and quantitative PCR. Total RNA was isolated from tissues, adipocytes, and macrophages with TRI Reagent (SigmaAldrich). RNA integrity was controlled by denaturing agarose gel electrophoresis. Only nondegraded RNA samples were used for cDNA synthesis, as described previously (46). Sorting of ATMs for Ki67 antigen required prior fixation with PFA, therefore, the RNeasy FFPE Kit (QIAGEN) was used to extract RNA from Ki67 ${ }^{-}$and Ki67 ${ }^{+}$ATMs. The ViiA 7 (Thermo Fisher Scientific) quantitative PCR platform was used for analysis, and gene expression values were expressed as relative mRNA levels according to dCt and ddCt methods, using Actb and Ppia (also known as Cypa) as reference genes for murine samples and $A C T B$ and PPIA as reference genes for human samples. The primer sequences are provided in Supplemental Table 2.

Supplemental methods. The assessment of glucose homeostasis in mice, TEM of ATMs, analysis of NPFF distribution in pancreas, isolation of resident macrophages, cell culturing of J774A.1 macrophages, Western blot analysis, cell-based ELISA, generation of transgenic mice and macrophages, NPFFR2-binding assay, intracellular cAMP measurement, and Affymetrix gene expression analysis are described in the Supplemental Methods. See complete unedited blots in the supplemental material. 
Statistics. All data represent the mean \pm SEM. For statistical analysis, an unpaired, 2-tailed Student's $t$ test, 1-way ANOVA with Dunnett's post-hoc test for multiple comparisons, or 2-way ANOVA was applied as appropriate. A $P$ value of less than 0.05 was considered significant. GraphPad Prism 5 (GraphPad Software) and MATLAB (MathWorks) statistical software programs were used.

Study approval. All animal experiments were carried out in accordance with local laws and were approved by local authorities (State of Baden Württemberg, 1206; Chang Gung University, CGU13-014; the IACUC of the University of Wisconsin; National Research Institute for Radiobiology and Radiohygiene Budapest; the University of Strasbourg, PEI/001/2073-6/2014; and the IACUC of the William S. Middleton Memorial Veterans Hospital, 02963.02). Human studies were approved by the local ethics commissions (University of Ulm, 121/16 22050 and 112/2003; University of Tennessee Health Science Center Institutional Review Board, 10-00848-XM; and the University of Valencia, PI 12/01978). None of the patients had clinical symptoms of systemic inflammation, and all provided written informed consent prior to their participation in the study. The patients were identified by numbers.

\section{Author contributions}

JCC, JSL, LB, JT, FS, JTR, EMS, DWL, and CMS provided methodology and designed experiments. SFHW, ACH, YTL, GA, HA, LB, JT, ICG, SN, JMI, SP, RQ, SA, and JAB performed experiments and analyzed data. TR conceived the project, designed experiments, and wrote the manuscript.

\section{Acknowledgments}

This study was supported by the European Commission Horizon 2020 Research and Innovation Program; a MarieCurie-Skłodowska Individual Fellowship (655598, to TR); the
German Research Fund (DFG, RO 4856/1-1, to TR); the German Academic Exchange Service DAAD (to SFHW); the Institute of Comparative Molecular Endocrinology (University of Ulm); the NIH (UC4 DK104155, to ICG); and the Juvenile Diabetes Research Foundation (47-2013-520, to ICG). FS, RQ, and SA were supported by the CNRS, Université de Strasbourg and the Laboratoire d'Excellence (LABEX) ANR-10-LABX-0034 Medalis. The laboratory of DWL is supported in part by the NIH (AG050135) and a Glenn Foundation Award for Research in the Biological Mechanisms of Aging. The study with Rnf128-KO and control mice was supported using facilities and resources from the William S. Middleton Memorial Veterans Hospital, and this work does not represent the views of the Department of Veterans Affairs or the United States Government. This work was funded in part by the National Cancer Institute (NCI), NIH, Comprehensive Cancer Center Support (CI P30 CA014520-UW, to CMS). The authors thank Susanne Schmidt, Svetlana Wostrezow, and Andriy Rebryk (University of Ulm, Germany) and Zita Pöstényi (NIRH, Budapest, Hungary) for their technical help; Jelena Pistolic and Vladimir Benes (Genomics Core Facility, European Molecular Biology Laboratory [EMBL], Heidelberg, Germany) for assistance with Affymetrix data processing; Marie-France Champy (INSERM, Illkirch, France) for help with metabolic studies; and Kenneth McCreath for critical reading of the manuscript. This study is dedicated to the memory of Prof. Dr. Pál Kertai (1927-2016); "Their leaves will not wither, nor their fruit fail."

Address correspondence to: Tamás Röszer. Institute of Comparative Molecular Endocrinology, Center of Biomedical Research, University of Ulm, Science Park I, 89081 Helmholtzstrasse 8.1, Ulm, Germany. Phone: 49.731.50.32630; Email: tamas.roeszer@ uni-ulm.de.
1. Boutens L, Stienstra R. Adipose tissue macrophages: going off track during obesity. Diabetologia. 2016;59(5):879-894.

2. Rosen ED, Spiegelman BM. What we talk about when we talk about fat. Cell. 2014;156(1-2):20-44.

3. Weisberg SP, McCann D, Desai M, Rosenbaum M, Leibel RL, Ferrante AW. Obesity is associated with macrophage accumulation in adipose tissue. J Clin Invest. 2003;112(12):1796-1808.

4. Amano SU, et al. Local proliferation of macrophages contributes to obesity-associated adipose tissue inflammation. Cell Metab. 2014;19(1):162-171.

5. Röszer T. Understanding the Mysterious M2 Macrophage through Activation Markers and Effector Mechanisms. Mediators Inflamm. 2015;2015:816460.

6. Quillet R, Ayachi S, Bihel F, Elhabazi K, Ilien B, Simonin F. RF-amide neuropeptides and their receptors in Mammals: Pharmacological properties, drug development and main physiological functions. Pharmacol Ther. 2016;160:84-132.

7. Murase T, Arima H, Kondo K, Oiso Y. Neuropeptide FF reduces food intake in rats. Peptides. 1996;17(2):353-354.

8. Lefrere I, et al. Neuropeptide AF and FF modulation of adipocyte metabolism. Primary insights from functional genomics and effects on beta-adrenergic responsiveness. J Biol Chem. 2002;277(42):39169-39178.

9. Herrera-Herrera ML, Salazar-Olivo LA. RFamide neuropeptides inhibit murine and human adipose differentiation. Biochem Biophys Res Commun. 2008;377(1):29-34.

10. Bonini JA, et al. Identification and characterization of two $\mathrm{G}$ protein-coupled receptors for neuropeptide FF. J Biol Chem. 2000;275(50):39324-39331.

11. van Harmelen V, et al. Effects of pain controlling neuropeptides on human fat cell lipolysis. Int JObes (Lond). 2010;34(8):1333-1340.

12. Hunt SC, et al. Polymorphisms in the NPY2R gene show significant associations with BMI that are additive to FTO, MC4R, and NPFFR2 gene effects. Obesity (Silver Spring). 2011;19(11):2241-2247.

13. Sundblom DM, Panula P, Fyhrquist F. Neuropeptide FF-like immunoreactivity in human plasma. Peptides. 1995;16(2):347-350.

14. Simonin F, et al. RF9, a potent and selective neuropeptide FF receptor antagonist, prevents opioid-induced tolerance associated with hyperalgesia. Proc Natl Acad Sci US A. 2006;103(2):466-471.

15. Watschinger K, et al. Tetrahydrobiopterin and alkylglycerol monooxygenase substantially alter the murine macrophage lipidome. Proc Natl Acad
Sci U S A. 2015;112(8):2431-2436.

16. Tokuoka SM, Kita Y, Shindou H, Shimizu T. Alkylglycerol monooxygenase as a potential modulator for PAF synthesis in macrophages. Biochem Biophys Res Commun. 2013;436(2):306-312.

17. Martinez FO, Gordon S, Locati M, Mantovani A. Transcriptional profiling of the human monocyte-to-macrophage differentiation and polarization: new molecules and patterns of gene expression. JImmunol. 2006;177(10):7303-7311.

18. Su S, et al. miR-142-5p and miR-130a-3p are regulated by IL- 4 and IL-13 and control profibrogenic macrophage program. Nat Commun. 2015;6:8523.

19. Wen AY, Sakamoto KM, Miller LS. The role of the transcription factor CREB in immune function. J Immunol. 2010;185(11):6413-6419.

20. Lumeng CN, Bodzin JL, Saltiel AR. Obesity induces a phenotypic switch in adipose tissue macrophage polarization. J Clin Invest. 2007;117(1):175-184.

21. Lawrence T, Natoli G. Transcriptional regulation of macrophage polarization: enabling diversity with identity. Nat Rev Immunol. 2011;11(11):750-761.

22. Hanson EM, Dickensheets H, Qu CK, Donnelly RP, Keegan AD. Regulation of the dephosphorylation of Stat6. Participation of Tyr-713 in the interleukin-4 receptor alpha, the tyrosine phos- 
phatase SHP-1, and the proteasome. J Biol Chem. 2003;278(6):3903-3911.

23. Rusinova I, et al. Interferome v2.0: an updated database of annotated interferon-regulated genes. Nucleic Acids Res. 2013;41(Database issue):D1040-D1046.

24. Sahoo A, Alekseev A, Obertas L, Nurieva R. Grail controls Th2 cell development by targeting STAT6 for degradation. Nat Commun. 2014;5:4732.

25. Soucie EL, et al. Lineage-specific enhancers activate self-renewal genes in macrophages and embryonic stem cells. Science. 2016;351(6274):aad5510.

26. Jenkins SJ, et al. Local macrophage proliferation, rather than recruitment from the blood, is a signature of TH2 inflammation. Science. 2011;332(6035):1284-1288.

27. Zheng C, et al. CD11b regulates obesityinduced insulin resistance via limiting alternative activation and proliferation of adipose tissue macrophages. Proc Natl Acad Sci U S A. 2015;112(52):E7239-E7248.

28. Menéndez-Gutiérrez MP, et al. Retinoid X receptors orchestrate osteoclast differentiation and postnatal bone remodeling. JClin Invest. 2015;125(2):809-823.

29. Aziz A, Soucie E, Sarrazin S, Sieweke MH. $\mathrm{MafB} / \mathrm{c}-\mathrm{Maf}$ deficiency enables self-renewal of differentiated functional macrophages. Science. 2009;326(5954):867-871.

30. Anderson KJ, Russell AP, Foletta VC. NDRG2 promotes myoblast proliferation and caspase $3 / 7$ activities during differentiation, and attenuates hydrogen peroxide - But not palmitate-induced toxicity. FEBS Open Bio. 2015;5:668-681.

31. Luan Y, Lengyel P, Liu CJ. p204, a p200 family protein, as a multifunctional regulator of cell proliferation and differentiation. Cytokine Growth Factor Rev. 2008;19(5-6):357-369.

32. Choubey D, Panchanathan R. Interferon-inducible Ifi200-family genes in systemic lupus erythematosus. Immunol Lett. 2008;119(1-2):32-41.

33. Liu L, Wang Y, Fan Y, Li CL, Chang ZL. IFN-gamma activates CAMP/PKA/CREB signaling pathway in murine peritoneal macrophages. J Interferon Cytokine Res. 2004;24(6):334-342.

34. Sato S, Yanagawa Y, Hiraide S, Iizuka K. Cyclic AMP signaling enhances lipopolysaccharide sensitivity and interleukin-33 production in RAW264.7 macrophages. Microbiol Immunol. 2016;60(6):382-389.

35. Tanabe K, Kozawa O, Iida H. cAMP/PKA enhances interleukin-1 $\beta$-induced interleukin- 6 synthesis through STAT3 in glial cells. Cell Signal. 2016;28(1):19-24

36. Sundblom DM, Kalso E, Tigerstedt I, Wahlbeck K, Panula P, Fyhrquist F. Neuropeptide FF-like immunoreactivity in human cerebrospinal fluid of chronic pain patients and healthy controls. Peptides. 1997;18(7):923-927.

37. Burlet-Schiltz O, et al. Identification of neuropeptide FF-related peptides in human cerebrospinal fluid by mass spectrometry. FEBS Lett. 2002;532(3):313-318.

38. Sun YL, et al. The anti-inflammatory potential of neuropeptide FF in vitro and in vivo. Peptides. 2013;47:124-132.
39. Jhamandas JH, Goncharuk V. Role of neuropeptide FF in central cardiovascular and neuroendocrine regulation. Front Endocrinol (Lausanne). 2013;4:8.

40. Sohn JW. Network of hypothalamic neurons that control appetite. BMB Rep. 2015;48(4):229-233.

41. Lee EB, Kim A, Kang K, Kim H, Lim JS. NDRG2-mediated Modulation of SOCS3 and STAT3 Activity Inhibits IL-10 Production. Immune Netw. 2010;10(6):219-229.

42. Driessler F, Venstrom K, Sabat R, Asadullah K, Schottelius AJ. Molecular mechanisms of interleukin-10-mediated inhibition of NF-kappaB activity: a role for 550 . Clin Exp Immunol. 2004;135(1):64-73.

43. Zhang K, et al. Mndal, a new interferon-inducible family member, is highly polymorphic, suppresses cell growth, and may modify plasmacytoma susceptibility. Blood. 2009;114(14):2952-2960.

44. Dauffy J, Mouchiroud G, Bourette RP. The interferon-inducible gene, Ifi204, is transcriptionally activated in response to M-CSF, and its expression favors macrophage differentiation in myeloid progenitor cells. J Leukoc Biol. 2006;79(1):173-183.

45. Bourette RP, Mouchiroud G. The biological role of interferon-inducible $\mathrm{P} 204$ protein in the development of the mononuclear phagocyte system. Front Biosci. 2008;13:879-886.

46. Röszer T, et al. Autoimmune kidney disease and impaired engulfment of apoptotic cells in mice with macrophage peroxisome proliferator-activated receptor gamma or retinoid $\mathrm{X}$ receptor alpha deficiency. JImmunol. 2011;186(1):621-631. 